\title{
Biological pattern and transcriptomic exploration and phylogenetic analysis in the odd floral architecture tree: Helwingia willd
}

\author{
Cheng Sun ${ }^{\dagger}$, Guoliang Yu', Manzhu Bao, Bo Zheng and Guogui Ning ${ }^{*}$
}

\begin{abstract}
Background: Odd traits in few of plant species usually implicate potential biology significances in plant evolutions. The genus Helwingia Willd, a dioecious medical shrub in Aquifoliales order, has an odd floral architecture-epiphyllous inflorescence. The potential significances and possible evolutionary origin of this specie are not well understood due to poorly available data of biological and genetic studies. In addition, the advent of genomics-based technologies has widely revolutionized plant species with unknown genomic information.

Results: Morphological and biological pattern were detailed via anatomical and pollination analyses. An RNA sequencing based transcriptomic analysis were undertaken and a high-resolution phylogenetic analysis was conducted based on single-copy genes in more than 80 species of seed plants, including $\mathrm{H}$. japonica. It is verified that a potential fusion of rachis to the leaf midvein facilitates insect pollination. RNA sequencing yielded a total of 111450 unigenes; half of them had significant similarity with proteins in the public database, and 20281 unigenes were mapped to 119 pathways. Deduced from the phylogenetic analysis based on single-copy genes, the group of Helwingia is closer with Euasterids II and rather than Euasterids, congruent with previous reports using plastid sequences.
\end{abstract}

Conclusions: The odd flower architecture make H. Willd adapt to insect pollination by hosting those insects larger than the flower in size via leave, which has little common character that other insect pollination plants hold. Further the present transcriptome greatly riches genomics information of Helwingia species and nucleus genes based phylogenetic analysis also greatly improve the resolution and robustness of phylogenetic reconstruction in $\mathrm{H}$. japonica.

Keywords: Biological pattern, Helwingia Willd, Phylogenetic analysis, Transcriptome

\section{Background}

Helwingia Willd (Helwingia japonica), blooming from April to May and fruiting from August to October, is a dioecious shrub in Helwingiaceae. Currently, eight species were documented and five of them were discovered to distribute in China. It possesses high medical value, such as antibacterial, anti-inflammatory and blood lipid reducing effects [1]. The Aquifoliales, to which $H$. Willd belongs, have attracted researchers' exceptional interest for their evolutionary histories. The floral architecture of Helwingia

\footnotetext{
* Correspondence: whnobleman2004@yahoo.com.cn

'Equal contributors

Key laboratory of Horticultural Plant Biology, Ministry of Education, College of Horticulture and Forestry Sciences, Huazhong Agricultural University, Wuhan 430070, P. R. China
}

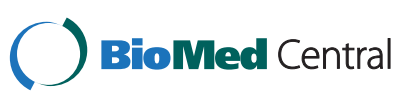

(c) 2014 Sun et al.; licensee BioMed Central Ltd. This is an Open Access article distributed under the terms of the Creative Commons Attribution License (http://creativecommons.org/licenses/by/2.0), which permits unrestricted use, distribution, and reproduction in any medium, provided the original work is properly credited. The Creative Commons Public Domain Dedication waiver (http://creativecommons.org/publicdomain/zero/1.0/) applies to the data made available in this article unless otherwise stated. species significantly differ from all other plants. The inflorescences of both sexes are borne on the laminae of scale and foliage leaves [2]. However the potential biology significances and the evolutionary origin of this odd floral architecture are not well understood due to poorly available data of biological and genetic studies in H. Willd. The genomic sequences and trancriptomic information can hardly be found in public databases.

The advent of genomics-based technologies has revolutionized the past sequencing me thod, and transcriptome sequencing is an alternative way to rich the genome information. The newly developed high throughput sequencing technology is a powerful and cost-efficient tool for advanced research in many areas, including genome resequencing, micro-RNA expression profiling, and especially 
de novo transcriptome sequencing for non-model organisms [3-5]. Over the past years, Next-Generation Sequencing (NGS) has greatly accelerated our understanding of the complexity of gene expression, regulation and networks in both model and non-model organisms [6-9].

The current phylogenetic studies in plants are dominated by sequencing the plastid and/or nuclear ribosomal DNA [10]. However, the markers from plastid genome or ribosomal DNA have limitations in phylogenetic analysis at the high taxonomic levels [11]. Phylogenetic analysis based on Low-copy nuclear genes has a great potential to complement cpDNA/nrDNA based analysis, and greatly improves the resolution and robustness of phylogenetic reconstruction at all taxonomic levels [12]. Large-scale transcriptome sequencing has the potential utility in functional genes exploration, and it also provides rich information for fine phylogenetic analysis.

In the present study, the biological and morphological pattern is illustrated in detail and the transcriptome of $H$. japonica is firstly characterizated utilizing Illumina paired-end sequencing technology based on the Nonnormalized cDNA of the leaves and flowers. A biological significance of odd floral architecture in Helwingia is suggested based on biological and morphological analysis. Combining the collected single-copy genes of more than 80 species, the phylogenetic analysis were undertaken and the preliminary conclusion of $H$. Willd 's evolutionary location was evaluated via comparative analysis to two classified methods, the Cronquist [13] and the APG III [14,15] system, respectively. The conclusion robustly confirms that those single-copy genes can be exploited in the evolutionary and classification research.

\section{Results}

\section{Morphological and biological pattern of $H$. Willd}

Five species of $H$. Willd growing in China show that all of them have epiphyllous inflorescences. During one growing season, the plant produces two type of leaves, the sterile foliage leaves (i.e., not inflorescence-bearing) and the fertile leaves (i.e., inflorescence-bearing). Of all the three presented species, the midrib color, until the inflorescence, of the fertile leaves is darker than that at the leaf tip. Simultaneously, the midrib between the petiole and the inflorescence is wider and thicker than the leaf tip (Figure 1A-C). The inflorescences of the fertile leaves are usually borne singly on the adaxial side of the midrib in the lower half of the lamina. They are imperfect flower, with only male or female reproductive organs. The flower have trimerous to pentamerous organs, possessing three to five petals, three to five calyx teeth and one flat floral disk. The male inflorescence of $H$. chinensis was a simple umbel composed of purplegreen flowers; each of them has a small calyx with a two to ten-millimeter long pedicel, three to five petals and three to five stamens (Figure 1D). Like other common plants, transverse sections analysis of male flower show that it has butterfly-like anthers, and that its clinandrium have three layers, including the tapetum, mesoderm and epidermis, arranging from inside to outside, respectively (Figure 1E). The female inflorescence grows one to three flowers lacking of the stamens, they were nearly sessile or with short pedicels (less than $2 \mathrm{~mm}$ long), and their stigma have three to five divided carpels (Figure 1F). The ovary is inferior and unilocular with one pendant ovule. The stigma divides and the pollen tube grows along the guided tissue. The longitudinal section of its oval-shaped ovule indicated that the ovule had a thick integument and an oval-shaped nucellar (Figure 1G). The vascular anatomy of the mature leaf shows that a separated, adaxial vascular bundle departs from the leaf trace in the base of the petiole and leads to the inflorescence, in the mature fertile leaf. In this case, an abaxial collateral bundle curves around and opposes an adaxial collateral bundle (Figure 1I). The abaxial component continues as the midrib vascular bundle into the leaf tip (Figure 1H).

Continuous investigation during the whole flowering times verified that $H$. Willd was insect pollinated. Stinkbug (Cletus punctiger) and ant were the two most common types of insects visiting the flowers (Figure 1J-K). The leaves of Helwingia gave both the ant and stinkbug enough space to stand on when they were visiting the flowers. This phenomenon proved that the evolutionary odd floral architecture in Helwingiaceae made it greatly adapted to insect pollination.

\section{Comparison with the related species in traditional classification}

The Helwingia genus was in Cornaceae (Table 1) according to the Cronquist traditional classification system [13]. The species in Helwingia genera had the similar biological and morphological pattern in flower characteristics, i.e. same number of the flower-merous and inferior ovary (Table 1). Such main characters also are observed in other genus species within Cornaceae. While it is not typical that there are many consistency characters among those species in the order level except for the ovary inferior trait (Table 1). Sub-class Rosidae Cornales include Celastrales, Apliales, Rosales and other orders, most species from them share the same number of flower-merous approximately. Compared with species in Asteridae, related to Rosidae, species in Asteridae tend to have superior ovary. There is no obvious similarity among those species at this level.

\section{Paired-end sequencing and de novo assembly}

A total of 40833338 raw reads with the length of $100 \mathrm{bp}$ were generated from a $200 \mathrm{bp}$ insert library using Illumina 

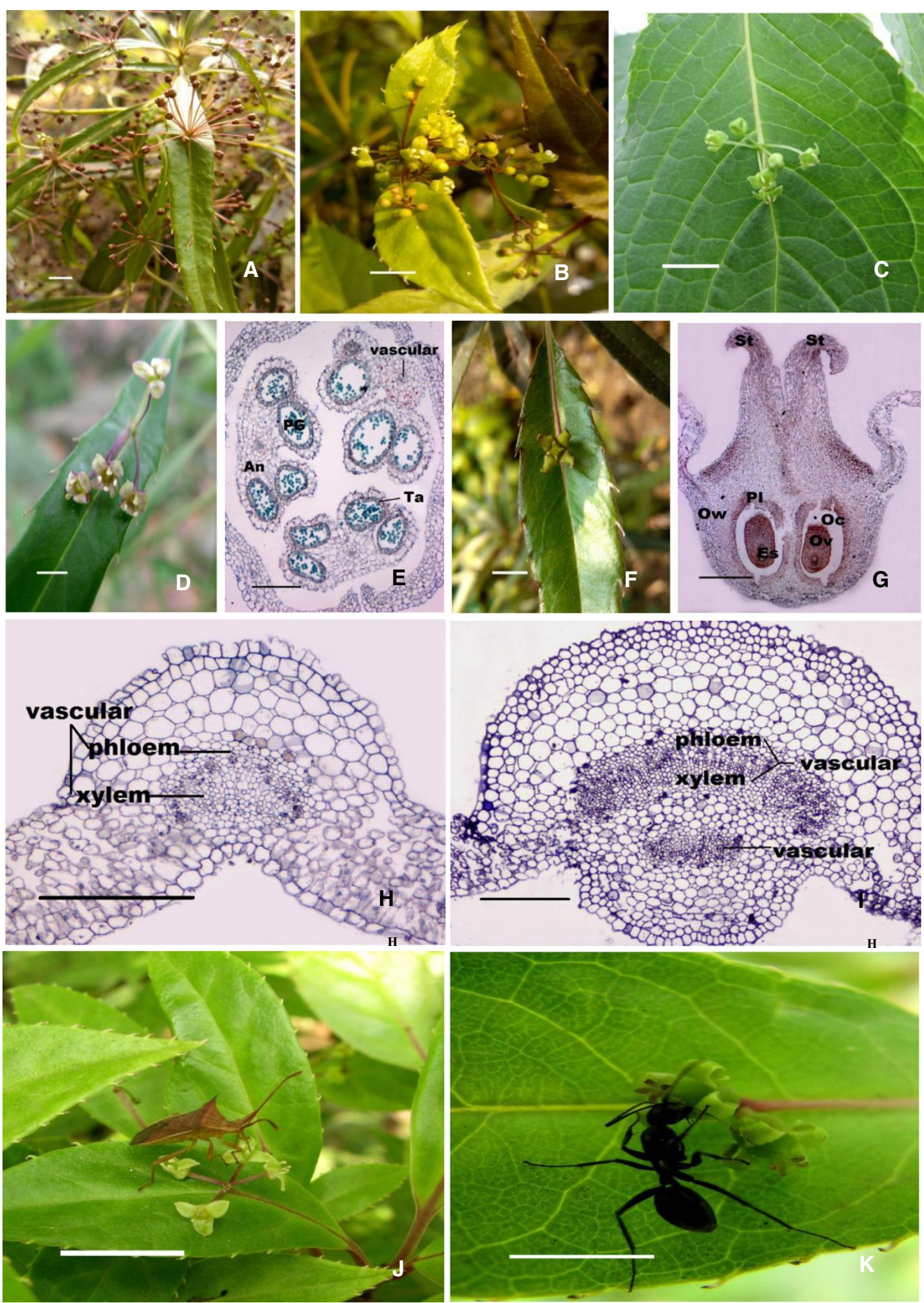

Figure 1 (See legend on next page.) 
(See figure on previous page.)

Figure 1 Morphological and Biological pattern of H. Willd. (A-C) Flower of H. chinensis, H. chinensis Batal. var. crenata and $\mathrm{H}$. japonica respectively; (bar $1 \mathrm{~cm})$. (D-E) Male Flowers of $\mathrm{H}$. Chinensis and its' transverse section, in which purple green flowers growing on midvein of leaf blade; (D: 5 mm; E: bar $0.1 \mathrm{~mm}$ ). (F-G) Female Flower of H. Chinensis and its' longitudinal section, in which the stigma was four divided; (F: bar $5 \mathrm{~mm}$; G: bar $1 \mathrm{~mm}$ ). (H) Transverse section of the top part of H. japonica leaf, in which one vascular is observed; (bar $1 \mathrm{~mm})$ (I). Transverse section of the based part of $\mathrm{H}$. japonica leaf, in which two vascular bundles are observed (obvious xylem, cambium and phloem are indicated); (bar $1 \mathrm{~mm}$ ) (J-K). Stink Bug and Ant are visiting the Helwingia flowers in pollination.(bar $1 \mathrm{~cm}$ ) Note: Po Pollen sac; An Anther; PG Pollen Granule; Ta Tapetum. OW Ovary Wall; Ov Ovule; PI Placenta; OC Locule; ES Embryo Sac; St Stigma.

paired-end sequencing method. The raw reads were cleaned by removing adaptor sequences, empty reads, and low-quality sequences. On high-quality cleaned reads, a total of 928284 contigs, ranging from 50 to $4732 \mathrm{bp}$, were assembled with an average length of $127 \mathrm{bp}$ and an N50 length of $99 \mathrm{bp}$. The contigs were then joined into scaffolds, based on paired-end information using " $N$ " to represent unkown nucleotides between each two contigs. 228299 scaffolds were obtained with an average length of $265 \mathrm{bp}$ (Table 2). The scaffold, ranging from 100 to 7239 bp, with an N50 length of 308 bp. Afterward, we used those paired-end reads again to fill the scaffold gaps to obtain unigenes with fewest Ns and could not be extended on either end. Finally the de novo assembly yields 111450 unigenes (Figure 2) with an average length of $400 \mathrm{bp}$ and a total length of $44.6 \mathrm{Mb}$, in which the N50 length was $420 \mathrm{bp}$. The length of assembled unigenes ranged from 200 to $7246 \mathrm{bp}$. Among these unigenes, 21698 (19.47\%) were greater than $500 \mathrm{bp}$ long and 4685 (4.20\%) were longer than $1 \mathrm{~kb}$ (Additional file 1). In addition, a total of 94406 (84.71\%) unigenes showed no gap (data were not shown). The best-aligning results from unigenes quality evaluation and CDS prediction showed that 54853 out of 111450 unigenes $(49.22 \%)$ had a BLAST

Table 1 Comparison of Helwingiacaea and related species under Cronquist system (1981)

\begin{tabular}{|c|c|c|c|c|c|c|c|c|c|c|}
\hline \multirow[t]{2}{*}{ Class } & \multirow[t]{2}{*}{ Order } & \multirow[t]{2}{*}{ Family } & \multirow[t]{2}{*}{ Genus } & \multirow[t]{2}{*}{ Species } & \multicolumn{6}{|c|}{ Characteristics } \\
\hline & & & & & $\begin{array}{l}\text { Flower } \\
\text { unisexual }\end{array}$ & Calyx & Petal & Stamen & Ovary & Locules \\
\hline Rosidae & Cornales & Cornaceae & Helwingia & Helwingia japonica & Yes & $3-5$ & $3-5$ & $3-5$ & Inferior & $3-5$ \\
\hline Rosidae & Cornales & Cornaceae & Helwingia & Helwingia chinensis & Yes & $3-5$ & $3-5$ & $3-5$ & Inferior & $3-5$ \\
\hline Rosidae & Cornales & Cornaceae & Helwingia & $\begin{array}{l}\text { H. chinensis Batal.var. } \\
\text { crenata }\end{array}$ & Yes & $3-5$ & $3-5$ & $3-5$ & Inferior & $3-5$ \\
\hline Rosidae & Cornales & Cornaceae & Cornus & Cornus officinalis & No & 4 & 4 & 4 & Inferior & 2 \\
\hline Rosidae & Cornales & Cornaceae & Swida & Swida alba & No & 4 & 4 & 4 & Inferior & 2 \\
\hline Rosidae & Cornales & Cornaceae & Swida & Swida wilsoniana & No & 4 & 4 & 4 & Inferior & - \\
\hline Rosidae & Cornales & Cornaceae & Aucuba & Aucuba chinensis & Yes & 4 & 4 & 4 & - & 2 \\
\hline Rosidae & Cornales & Garryaceae & Dendrobenthamia & $\begin{array}{l}\text { Dendrobenthamia } \\
\text { japonica var. chinensis }\end{array}$ & No & - & - & - & Inferior & - \\
\hline Rosidae & Cornales & Alangiaceae & Alangium & Alangium salviifolium & No & $4-10$ & $4-10$ & $20-30$ & Inferior & 1 \\
\hline Rosidae & Cornales & Davidiaceae & Davidia & Davidia involucrata & Yes \& No & - & $2-3$ & $1-7$ & Inferior & $6-10$ \\
\hline Rosidae & Celastrales & Aquifoliaceae & Ilex & llex chinensis & Yes & $4-5$ & $4-5$ & 4 & Superior & 4 \\
\hline Rosidae & Apliales & Araliaceae & Aralia & Aralia chinensis L. & No & 5 & 5 & 5 & - & 5 \\
\hline Rosidae & Apliales & Araliaceae & Panax & Panax ginseng & No & 5 & 5 & 5 & - & 2 \\
\hline Rosidae & Rosales & Grossulariaceae & Ribesa & Ribes nigrum & No & 5 & 5 & 5 & Inferior & - \\
\hline Rosidae & Rosales & Grossulariaceae & Ribesa & Ribes davidii & Yes & 5 & 5 & 5 & - & 2 \\
\hline Asteridae & Plantaginales & Plantaginaceae & Antirrhium & Antirrhium majus L. & No & - & 1 & 4 & - & - \\
\hline Asteridae & Asterales & Asteraceae & Gerbera & Gerbera jamesonii & Yes \& No & - & - & 5 & Inferior & 1 \\
\hline Asteridae & Asterales & Asteraceae & Helianthus & Helianthus_annuum & Yes \& No & 2 & Combined & 5 & Inferior & 1 \\
\hline Asteridae & Solanales & Solanaceae & Solanum & Solanum tuberosum & No & 5 & 5 & 5 & Superior & - \\
\hline Asteridae & Solanales & Solanaceae & Solanum & Solanum lycopersicum & No & $5-7$ & $5-7$ & $5-7$ & Superior & 2 to several \\
\hline Dilleniidae & Ericales & Theaceae & Camellia & Camellia sinensis & No & 5 & $5-6$ & Several & Superior & 3 \\
\hline Dilleniidae & Ericales & Ericaceae & Rhododendron & Rhododendron ponticum & No & 5 & 5 & 10 & Superior & 10 \\
\hline
\end{tabular}


Table 2 Overview of the sequencing and assembly

\begin{tabular}{lllll}
\hline & N50 & Mean size & Total length & Total number \\
\hline Read & - & 90 & 3675000420 & 40833338 \\
Contig & 99 & 127 & 118117971 & 928284 \\
Scaffold & 308 & 265 & 60563756 & 228299 \\
Unigene & 420 & 400 & 44587120 & 111450 \\
\hline
\end{tabular}

homologous match against the public protein databases. For those unigenes had no hit in blast, the CDS were predicted by ESTScan. Finally, 59101 unigenes (53.03\%) were oriented.

\section{Function annotation and Gene Ontology classification}

Sequence similarity search indicated the retrieved proteins had the highest sequence similarities with given unigenes and a total of 54853 (49.22\%) were annotated in this manner. To classify the function of the predicted $H$. japonica genes, Blast $2 \mathrm{GO}$ successfully annotated 27775 sequences to 101010 GO terms out of the total 54853 sequences with BLAST matches. In many cases, multiple terms were assigned to the same unigene, this resulted in 50713 unigenes assigned to "biological process" category, 55542 to "cellular component" category and 34538 to "molecular function" category. Among the various biological process, "metabolic process" (13359, 26.34\%) and "cellular process" (12121, 23.90\%) were the most represented (Figure 3). The genes, involved in other important biological process such as response to stimulus and biological regulation, were also identified through GO annotations. Similarly, "cell" and "cell" part (both 18096, 32.58\%) were prominently represented, whereas almost no genes were assigned to "virion" or "extracellular region". Under the category of molecular function, "binding" (16719, 48.41\%) and "catalytic activity" (13906, 40.26\%) represented the majority of the category.

\section{Function classification by COG and KEGG}

To further evaluate the completeness of annotation process, all unigenes were aligned to the COG database. 11732 sequences were assigned to the COG classifications (Figure 4). Among the 25 COG categories, the cluster for "general function prediction only" (2908, 15.58\%) represented the largest group, followed by "replication recombination and repair" (1841, 9.87\%), "transcription" (1558, $8.35 \%)$ and "posttranslational modification, protein turnover and chaperones" (1424, 7.63\%), whereas only a few unigenes were assigned to "extracellular structures" and "nuclear structure". 1211 unigenes were assigned to "signal transduction mechanisms". Based on a comparison against the KEGG database using BlastX (e-values < 1.00E-05), out of the 111450 unigenes, 20281 unigenes (18.20\%) can be mapped with 119 metabolic pathways (Additional file 2). 12481 unigenes have enzyme commission (EC) numbers, and were assigned to the metabolic pathways. The pathways with the most representations of unique sequences were the metabolic pathways (4610, 22.73\%), followed by those related to plant-pathogen interaction $(1657,8.71 \%)$ and splicesome (1113, 5.49\%).

\section{Shared single-copy genes in the solexa transcriptome} assemblies and their Phylogenetic analysis

13 confirmed shared sing-copy or low-copy nuclear genes $[7,11]$ across the major lineages of angiosperm were used to screen their orthologs in 14 transcriptome assemblies, including the transcriptome assembly of $H$. japonica in

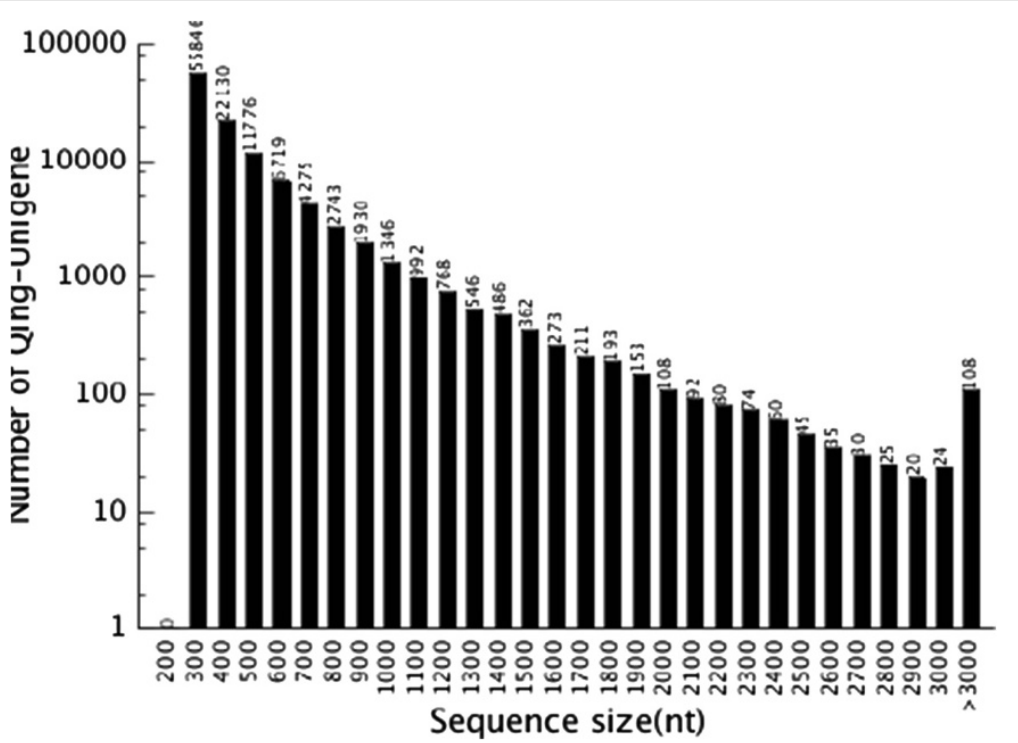

Figure 2 Assessment of assembly quality, which indicates distribution of unique mapped reads of the assembled unigenes. 

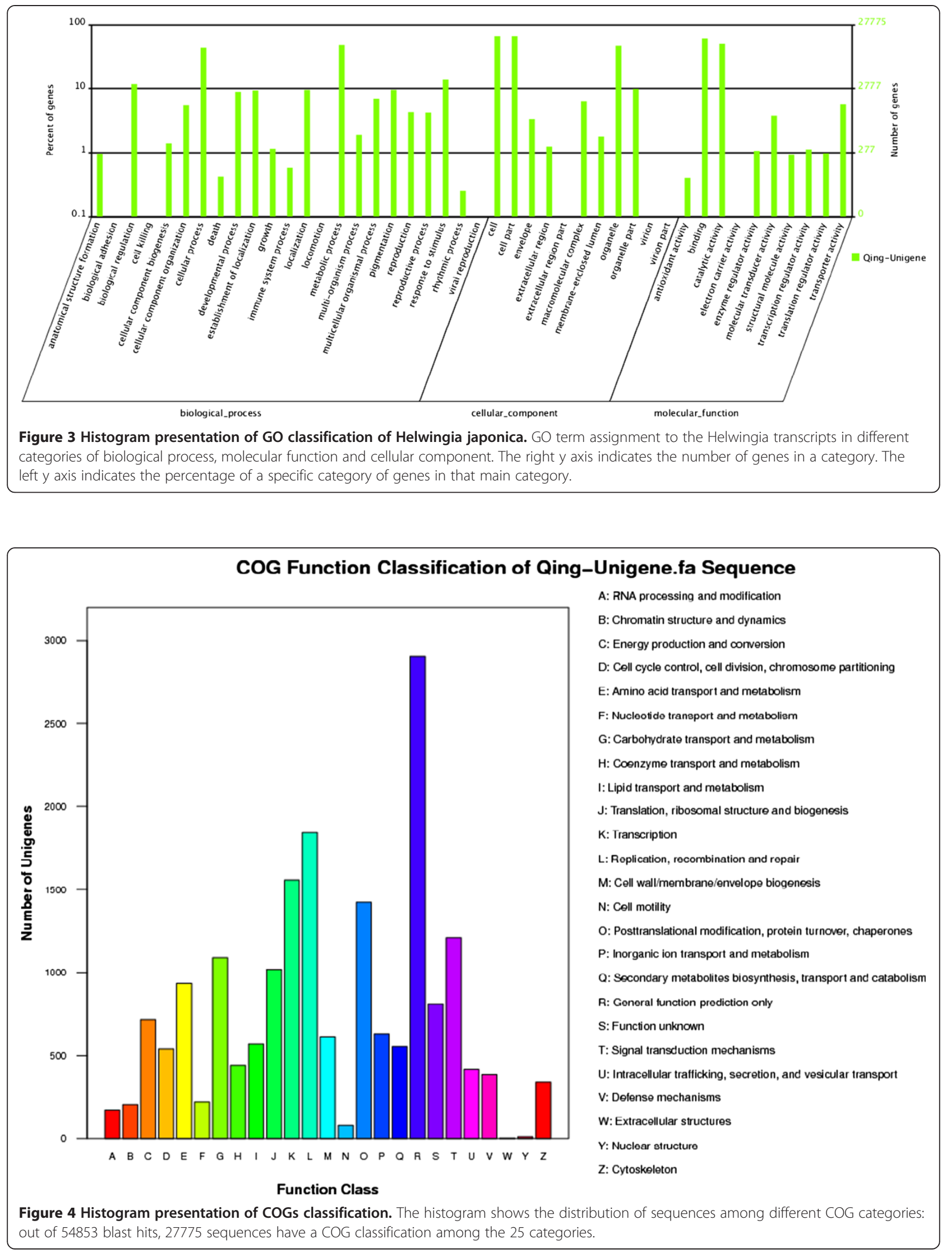
this study (Additional file 3). Finally, two close related species, H. japonica and Panax ginseng, together with the other twelve species, were used to perform phylogenomic analysis. These fourteen species contain at least 6 singlecopy homologous genes. For most of the thirteen singlecopy genes, only one sequence with high similarity to a certain gene was found, which indicates that they are single-copy in the sampled species. As for the sampled transcriptome that had multiple significant blast hits to a certain gene, the orthologs were identified using the bidirectional blast method. Orthologs of most of these genes were found in all these transcriptomes. The collected sequences were added to the dataset reported before $[7,11]$, and resulted in an 83-taxon nucleic acid sequence dataset containing 9159 characters (including gaps) (Additional file 4). The phylogeny was reconstructed using the maximum parisomny (MP) and maximum likelihood (ML) methods based on both nucleotide and amino acid sequences. The phylogenetic trees resulting from the two methods were similar to those inferred by the previously published studies based on those plastid sequences dataset [16-19] in topology and resolution though there were some differences in the placement of individual species between the MP and ML trees. Overall, the ML tree, with bootstrap values higher than 50 for most nodes in both nucleotide and amino acid based tree, shows improved resolution and increased bootstrap support compared to the MP tree (Figure 5).

\section{Classification of tested species using single or low copy nucleus genes and the similarity to APG III system}

The deduced phylogenetic trees are largely congruent between the MP and the ML methods. The resulting phylogeny topologies from ML tree shows improved resolution, which are greatly consistent to many previous phylogenetic studies based on plastid sequences at varying taxonomic levels (Figure 6). Our phylogenetic analysis based on the thirteen single-copy nuclear genes also supported that the group of Helwingia is closer with Euasterids II than Euasterids I (Figure 6). Our data also strongly suggests that the relationships between those clades of Asterids, including Ericales, Lamiids (ie. Euasterids I) and Campanulids (ie. Euasterids II), are sister relationships. The result of subgroups is also similarity to the APG III system that the Helwingia genus, belonging to Aquifoliales, is placed under the clade of Campanulids.

\section{Discussion}

Helwingia implicates a biological significance on Odd floral architecture

In $H$. Willd, the fusion of flower stalk to petiole might protect the flowers from rigid environments or shorten the distance of nutrition supplementation from leaves to flowers. In this study, the fusion pattern was carefully characterized by vascular anatomy. Floral adaptation to animal or abiotic pollination is considered to have been a key basis for the morphological diversification of angiosperms [23]. On common, for insect pollination, the animal size is usually much smaller than the flower size. Helwingia does not have big flowers, however their wide leaf blades provide the insects enough space to stand on when they visit the flowers, which further verifies how Helwingiaceae species present alternative pattern to adapt to insect pollination. In $H$. Willd the size of insects is much larger than that of flowers, so it is very important for Helwingiaceae to uphold the visiting insects with the leaves during pollination. Fossil evidence indicates that ants emerged in the Late Jurassic, 150 million years ago, while the flowering plants 100 million years ago [24,25]. Another Helwingia flower visiting insect - stinkbugs were assumed to emerge from the Late Triassic Epoch to the Middle Ages, but mostly in the Middle Ages [26]. Whether it is an evolution or an incident case to make the odd floral architecture, it gives us infinite cues.

\section{Transcriptome analysis riches genomics information of Helwingia species}

Prior to this study, the limited numbers of sequences (only 35 ESTs) were deposited in GenBank. Our transcriptomic analysis yielded over 111 thousands of unigenes that could be matched to known genes via BLAST search, and many of them are involved in leaf and floral development. Those "non-blastable" sequences, to some extend, are inherent to the following categories: the non-coding cDNA fraction, lineage-specific genes and fast-evolving genes. This relative fraction of unigenes that did not have any BLAST hits may be an integral part of genomic data [7]. The transcriptome analysis of $H$. japonica might be more effective to get lots of genomic data and more helpful to deep the related researches in Helwingia species.

Comparison of assembled gene models and functional annotation (GO, COG, KEGG) indicates that we have sampled an expansive and diverse expressed gene catalog representing a large proportion of the genes expressed in Helwingia. The Aquifoliales, which $H$. Willd belongs to, have attracted an great interest from evolutionary biologist. The presented transcriptomic data will be significantly important for further research on functional genomics.

\section{Transcriptomic data set effectively used to perform phylogenetic analysis}

Plant phylogenetic studies has been dominated by using the plastid sequences (e.g. matK, trnL-F and $r b c L$ ) and/or nuclear ribosomal DNA (18S, 26S, ITS and ETS) [10,27]. However, the markers from plastid genome or ribosomal DNA have limitations on phylogenetic utilities at high taxonomic levels [12]. Single or low-copy nuclear genes 


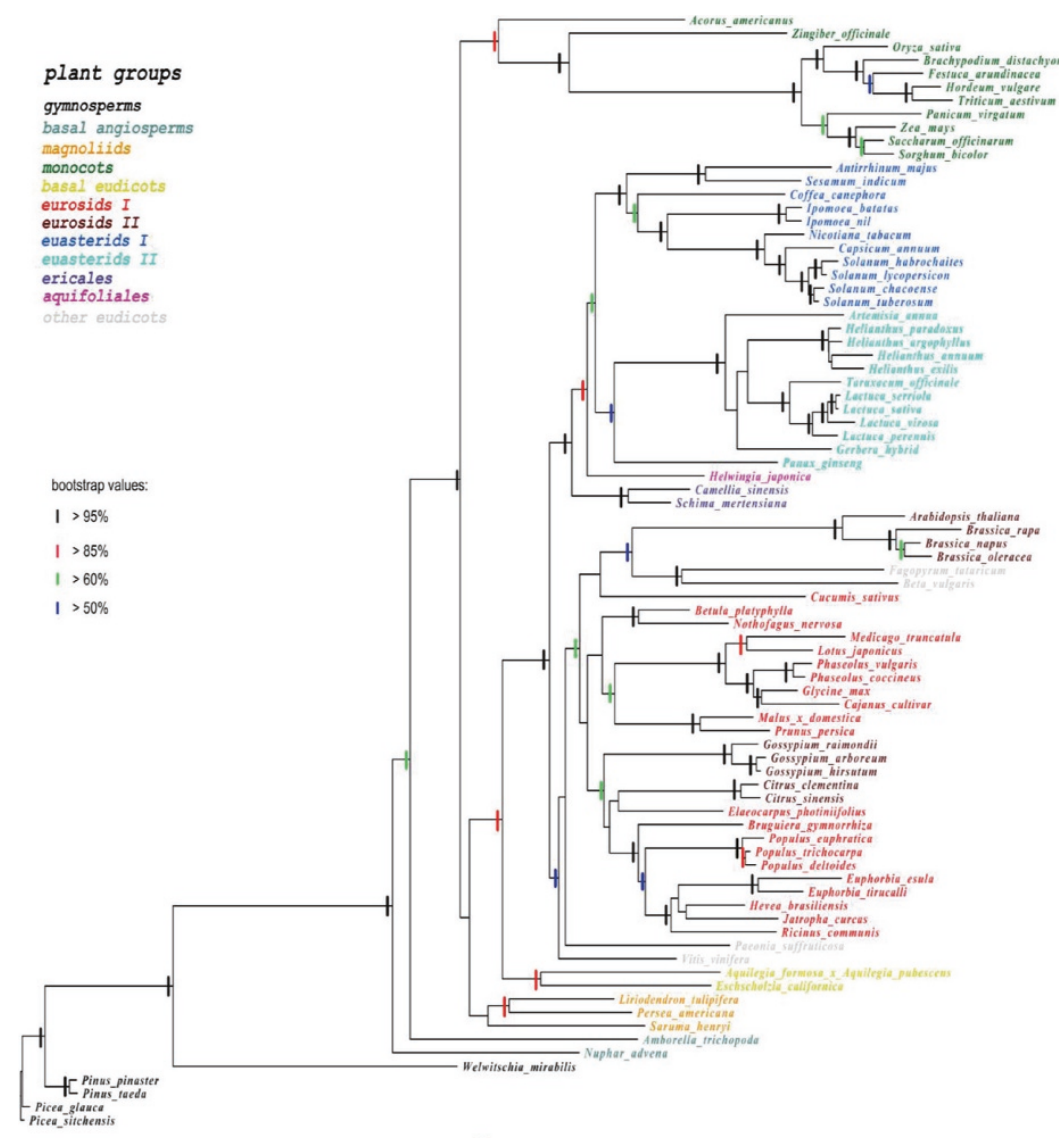

$\mathbf{A}^{0}$

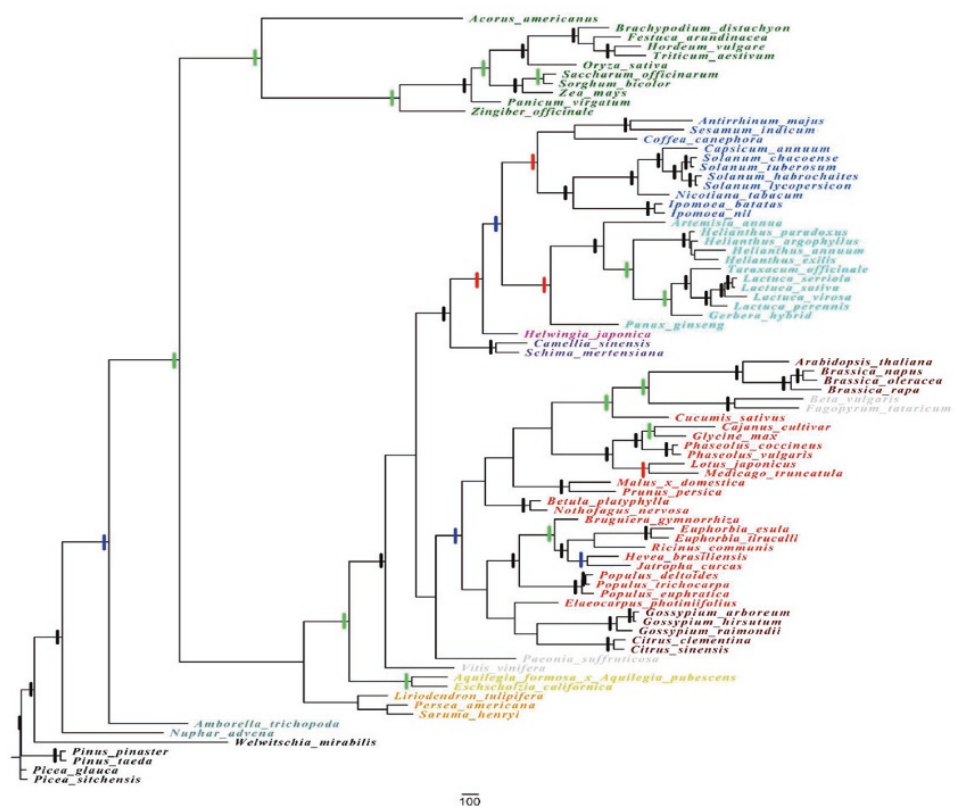

B

Figure $\mathbf{5}$ (See legend on next page.) 
(See figure on previous page.)

Figure 5 Trees inferred from the maximum maximum likelihood analysis (A) and maximum parsimony analysis (B) of nucleotide sequences of 13 single-copy genes. Picea sitchensis was used as the outgroup taxa for all analyses. Branch lengths are proportional to the number of expected nucleotide substitutions; scale bar corresponds to one substitution per hundred sites for the ML tree and to 100 changes for maximum parsimony tree. Non-parametric bootstrap values (greater than 50\%) are indicated by the colored bars placed on branches.

have a great potential, in compensation for cpDNA and nrDNA, to improve the resolution and robustness of phylogenetic reconstruction at all taxonomic levels. Transcriptomic data is a potential source of information for multigene phylogenetic analysis. Duarte et al. identified a set of single-copy genes shared between Arabidopsis, Populus, Vitis and Oryza [11]. Though many articles have reported about the evolutionary position of Helwingiaceae at varying taxonomic levels, none of them indicated the use of nuclear gene for phylogenetic analysis. Based on these single or low copy nuclear genes from more than 80 species, including Helwingiaceae, the phylogenetic tree inferred from the maximum parsimony and maximum likelihood analysis shown a higher resolution and node support. Our result is consistent with the privious hypothesis based on multiple plastid sequences.

\section{Phylogenetic analysis based on Helwingia nuclear genes is consistent with that based on plastid or ribosomal DNA sequences}

Comparative analysis among varied classified system indicated that the tradition classification, the Cronquist system, was not quite consistent with our phylogenetic analysis. Panax ginseng was placed in Rosidae according to the traditional classification, whereas our phylogenetic analysis showed that Panax ginseng was indeed close to H. japonica. H. Willd was classified to Cornaceae in the systematics of Cornaceae, which is suggested remarkably unsettled and controversial by some researchers [28]. Many genera had been added to or removed from Cornaceae with time [29,30]. Currently, phylogenetic analyses based on plastid or ribosomal DNA datasets have consistently supported that Aquifoliaceae (Ilex only), Helwingiaceae (Helwingia only), and Phyllonomaceae (Phyllonoma only) were sisters within Aquifoliales [16,19]. The nuclear genes-based phylogenetic analysis here gives another solid evidence on it and confirm the phylogenetic location of $H$. japonica, which also presents the same trends as Duarte's reports on some tested species using limited single or low-copy nuclear genes to perform phylogenetic analysis [11]. Additional, the result is also consistent with previou phylogenetic studies referred to Helwingiaceae based on plastid or ribosomal DNA [28,31]. Moreover, this presented subset produced well-resolved tree topologies similar to that inferred based on plastid or chloroplast sequences in many recent phylogenetic studies $[7,17]$. The phylogenetic tree presented in our study is somewhat similar with the circumscription of the genera of Helwingia in APG III (Angiosperm Phylogeny Group III) system. In APG III, the genera of Helwingia is placed within the

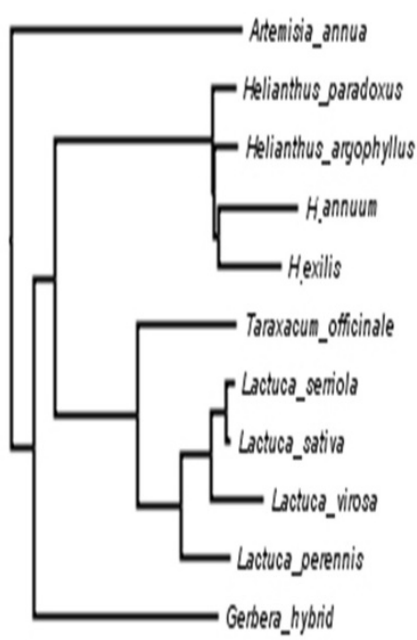

A
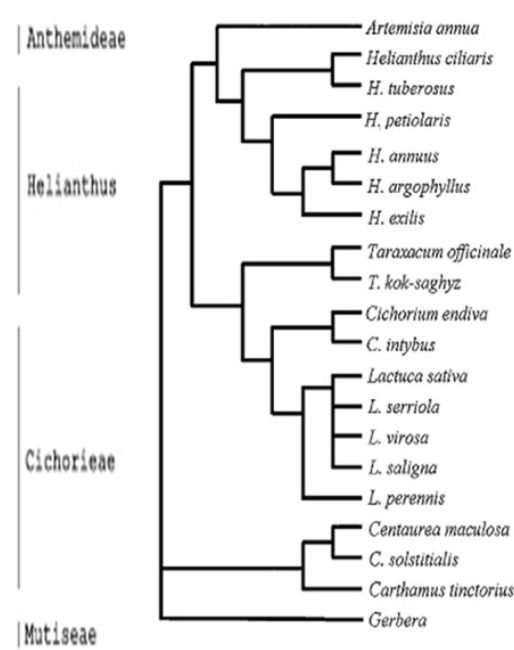

B

Figure 6 Comparative analyses between phylogenetic relationships nuclear genes and plastid sequences. (A) The interception of the phylogenetic relationships for the sampled lineages of Asteraceae from our ML tree based on 13-single copy nuclear genes. (B) Phylogenetic inferred relationships for the major lineages of Asteraceae based on ribosomal sequences from Rieseberg [20] Funk et al. [21], and Timme et al. [20-22]. 
order Aquifoliales, under the clade of Campanulids. In the MP and ML trees, Helwingia was both identified as more closely related to Euasterids II than to Euasterids I.

\section{Conclusions}

The present study characterized the biological and transcriptomic patterns of $H$. japonica, a rare but vital species for research of evolutionary mechanism. The morphological and pollinated characteristics were elucidated and a comprehensive transcriptome is firstly developed. About 111450 assembled transcripts were obtained, with a half of them matching to known proteins. In addition, a phylogenetic analysis based on nuclear single or low-copy genes was proceeded, which has a great potential to complement cpDNA and nrDNA based phylogenetic analysis and enhances the resolution of plant phylogenetic reconstruction at all taxonomic levels. The $H$. japonica transcriptome and the collected low-copy nuclear genes dataset from more than 80 species reported in our study will rich the genomic resources for Helwingia and Asterids order. It will also be a significant contribution towards reconstruction of the plant phylogenetic tree.

\section{Methods}

\section{Sample collection and preparation}

Tissue samples of $H$. Willd, were collected from mature plants growing in both the Wuhan Botanical Garden (Hubei Province) and Mt. Lushan (Jiangxi Province) in China. Standard procedures of paraffin section analysis were according to our previous described method [32]. The sections were observed and photographed under appropriate magnifications using a NIKON research microscope.

\section{RNA isolation and CDNA library preparation for transcriptome analysis}

Total RNA was isolated using TRIzol reagent (Invitrogen) according to the manufacturer's instructions. mRNA was purified using oligo (dT) magnetic beads, and then fragmented into small pieces using divalent cations under elevated temperature. The cleaved RNA fragments were transcribed into first-strand cDNA using reverse transcriptase and random hexamer-primers, followed by second-strand cDNA synthesis using DNA polymerase I and RNaseH. After end repairing, adapter ligations were conducted. The products were purified and enriched with PCR to create the final cDNA library. All the experiments were undertaken in the Beijing Genome Institute (BGI, Shenzhen, China).

\section{Sequencing and de novo assembly}

cDNA library was sequenced on an Illumina HiSeq2000 sequencing platform. The average size of inserts in the library was $200 \mathrm{bp}$, and it generates $100 \mathrm{bp}$ PE reads [4]. Image deconvolution and quality value calculations were performed using Illumina HCS 1.1 software. De novo assembly was carried out using SOAPdenovo (http://soap.genomics.org.cn/soapdenovo.html) with the default settings [33] except with $29 \mathrm{~K}$-mers after varied K-mers were firstly tested. Contigs without ambiguous bases were obtained by conjugating the K-mers in an unambiguous path. Next, SOAPdenovo connected the contigs using $\mathrm{N}$ to represent unknown sequences via the paired-end information to generate Scaffolds. Paired-end reads were used again for gap filling of scaffolds to get sequences with least Ns and cannot be extended on either end, which were finally defined as Unigenes. To obtain distinct gene sequences, the unigenes were clustered using the TGICL (TIGR Gene Indices Clustering) tools.

\section{Function annotations of transcriptome}

Unigenes were aligned with the NCBI Nr and Swiss-Prot protein databases using BLASTx [34] with an E-value cut-off of $10^{-5}$ to evaluate the quality and predicted CDS. Unigenes that did not have significant hits in these databases were scanned using ESTScan [35]. Blast2GO [36] was used to obtain GO (Gene ontology) terms according to molecular function, biological process and cellular component (http://www.geneontology.org) based on BLASTx hits against the NCBI Nr database. Annotation with the COG and KEGG [37] pathways were also performed using BLASTx against the COG database and the Kyoto Encyclopedia of Genes and Genomes database.

\section{Phylogenetic analysis}

For the phylogenetic analysis, a dataset of 13 single-copy genes from 69 taxa was collected from Duarte et al. [11]. The orthologs of these genes were screened by the BLAST search of corresponding Arabidopsis and Oryza genes in sixteen NGS transcriptomic datasets of core eduicots. Six of them were from Asterids: H. japonica, Panax genseng, Camellia sinensis, Schima mertensiana, Artemisia annua and Sesamum indicum. Sequences of orthologs of these 13 genes in 14 transcriptomes were added to the alignment. The whole set was checked for frame shifts and if present, then corrected by inserting $\mathrm{N}$ to recover in frame translation. Translated protein sequences for the orthologs were made using EMBOSS [35] and aligned using MUSCLE [38]. The corresponding coding DNA sequence alignment was calculated using the program RevTrans1.4 [39]. Poorly aligned positions were removed using Gblocks (http://molevol.cmima.csic.es/castresana/ Gblocks.html) with all options for a less stringent selection. Amino acid sequence alignments and nucleotide alignments were used to produce phylogenies using maximum parsimony (MP) and maximum likelihood (ML). The GTR + I + T model of nucleotide substitution were determined by the Akaike information criterion (AIC) in Modeltest ver. 3.7 [40]. The JTT model of amino acid substitutions was determined by the AIC in ModelGenerator 
for ML analysis. The Maximum Likelihood analysis was explored using PhyML 3.0 [41] for both to the amino acid and nucleotide data. The non-parametric bootstrap analysis was performed with 1000 replicates. The phylogenetic analysis using the Maximum Parsimony (MP) method was performed using PAUP* ver. 4. 0b8 [42]. The MP analysis involved a heuristic search using TBR branch swapping and 20 random addition replicates. Support for nodes was also evaluated with 1000 replicates of non-parametric bootstrapping.

\section{Additional files}

\section{Additional file 1: All of the assembled unigenes derived from \\ H. Willd.}

Additional file 2: The mapped metabolic pathways of part of unigenes derived from $\mathrm{H}$. Willd.

Additional file 3: The shared 13 sing-copy nuclear genes sequence from 83-taxon species.

Additional file 4: Alignment of 13 single copy genes sequence in 83-taxon species.

\section{Competing interests}

The authors declare that they have no competing interests.

\section{Authors' contributions}

CS and GY performed the experiments. CS drafted the manuscript. BZ, MB and $\mathrm{GN}$ finalized the paper. GN supervised the project. All authors read and approved the final manuscript.

\section{Acknowledgements}

This work was supported by grants from the Fundamental Research Funds for the Central Universities (2013PY038). We thank all the colleagues in our lab for constructive discussion and technical support, and Dr. Alex McCormac (Mambo-Tox Ltd., UK) for critical editing of the manuscript.

Received: 17 September 2013 Accepted: 19 June 2014 Published: 27 June 2014

\section{References}

1. Chen L, Li W-J, Yao M, Li X, Zhao B, Zhang T-L, Jia X, Gong J, Ni S-F: Overview of Pharmaceutical Research on Helwingia Willd. J Liaoning University TCM 2012, 14:116-117.

2. Dickinson TA, Sattler R: Development of the epiphyllous inflorescence of Helwingia Japonican (Heliwingiaceae). Amer J Bot 1975, 62:962-973.

3. Collins $\sqcup$, Biggs PJ, Voelckel C, Joly S: An approach to transcriptome analysis of non-model organisms using short-read sequences. Genome Inform 2008 21:3-14.

4. Fullwood MJ, Wei C-L, Liu E-T, Ruan Y: Next-generation DNA sequencing of paired-end tags (PET) for transcriptome and genome analyses. Genome Res 2009, 19:521-532.

5. Robertson G, Schein J, Chiu R, Corbett R, Field M, Jackman SD, Mungall K, Lee S, Okada HM, Qian JQ, Griffith M, Raymond A, Thiessen N, Cezard T, Butterfield YS, Newsome R, Chan SK, She R, Varhol R, Kamoh B, Prabhu AL, Tam A, Zhao YJ, Moore RA, Hirst M, Marra MA, Jones SJM, Hoodless PA, Birol I: De novo assembly and analysis of RNA-seq data. Nat Methods 2010, 7:909-912.

6. Russell JR, Bayer M, Booth C, Cardle L, Hackett CA, Hedley PE, Jorgensen L, Morris JA, Brennan RM, Russell JR: Identification, utilisation and mapping of novel transcriptome-based markers from blackcurrant (Ribes nigrum). BMC Plant Biology 2011, 11:147.

7. Logacheva MD, Kasianov AS, Vinogradov DV, Samigullin TH, Gelfand MS, Makeev VJ, Penin AA: De novo sequencing and characterization of floral transcriptome in two species of buckwheat (Fagopyrum). BMC Genomics 2011, 12:30.
8. Wei W, Qi X, Wang L, Zhang Y, Hua W, Li D, Lv H, Zhang X: Characterization of the sesame (Sesamum indicum L.) global transcriptome using Illumina paired-end sequencing and development of EST-SSR markers. BMC Genomics 2011, 12:451.

9. Zhang JA, Liang S, Duan JL, Wang J, Chen SL, Cheng ZS, Zhang Q, Liang X, Li YR: De novo assembly and characterisation of the transcriptome during seed development, and generation of genic-SSR markers in peanut (Arachis hypogaea L.). BMC Genomics 2012, 13:90.

10. Kelchner SA: The evolution of non-coding chloroplast DNA and its application in plant systematics. Ann Mo Bot Gard 2000, 87:482-498.

11. Duarte JM, Wall PK, Edger PP, Landherr LL, Ma H, Pires JC, Leebens-Mack J, Depamphilis CW: Identification of shared single copy nuclear genes in Arabidopsis, Populus, Vitis and Oryza and their phylogenetic utility across various taxonomic levels. BMC Evol Biol 2010, 10:61.

12. Hughes CE, Eastwood RJ, Bailey CD: From famine to feast? Selecting nuclear DNA sequence loci for plant species-level phylogeny reconstruction. Philos Trans R Soc Lond B Bio/ Sci 2006, 361:211-225.

13. Cronquist S: An Integrated System of Classification of Flowering Plants. New York, New York, USA: Columbia University Press; 1981.

14. Angiosperm Phylogeny Group: An update of the Angiosperm Phylogeny Group classification for the orders and families of flowering plants: APG III. Bot J Linn Soc 2009, 161:105-121.

15. Angiosperm Phylogeny Group: An update of the Angiosperm Phylogeny Group classification for the orders and families of flowering plants: APG II. Bot J Linn Soc 2003, 141:399-436.

16. Tank DC, Donoghue MJ: Phylogeny and phylogenetic nomenclature of the Campanulidae based on an expanded sample of genes and taxa. Syst Bot 2010, 35:425-441.

17. Moore MJ, Soltis PS, Bell CD, Burleigh JG, Soltis DE: Phylogenetic analysis of 83 plastid genes further resolves the early diversification of eudicots. Proc Natl Acad Sci 2010, 107:4623-4628.

18. Kane NC, Barker MS, Zhan SH, Rieseberg LH: Molecular evolution across the Asteraceae: micro- and macroevolutionary processes. Mol Biol Evol 2011, 28:3225-3235.

19. Soltis DE, Smith SA, Cellinese N, Wurdack KJ, Tank DC, Brockington SF, Refulio-Rodriguez NF, Walker JB, Moore MJ, Carlsward BS, Bell CD, Latvis M, Crawley S, Black C, Diouf D, Xi Z, Rushworth CA, Gitzendanner MA, Sytsma KJ, Qiu YL, Hilu KW, Davis CC, Sanderson MJ, Beaman RS, Olmstead RG, Judd WS, Donoghue MJ, Soltis PS: Angiosperm phylogeny: 17 genes, 640 taxa. Am J Bot 2011, 98:704-730.

20. Rieseberg LH: Hybridization in rare plants: insights from case studies in Cercocarpus and Helianthus. New York, New York, USA: Oxford University Press; 1991.

21. Funk VA, Bayer RJ, Keeley S, Chan R, Waston L, Gemeinholzer B, Schilling E, Panero JL, Baldwin BG, Garcia-Jacas N, Susanna A, Jansen RK: Everywhere but Antarcica: using a supertree to understand the diversity and distribution of the Compositae. Boil. Skr 2005, 55:343-374.

22. Timme RE, Kuehl JV, Boore $J$, Jansen RK: A comparative analysis of the Lactuca and Helianthus (Asteraceae) plastid genomes: identification of divergent regions and categorization of shared repeats. Am J of Botany 2007, 94:302-312.

23. Eriksson $\mathrm{O}$, Bremer B: Pollination systems, dispersal modes, life forms, and diversification rates in angiosperms families. Evolution 1992, 46:258-266.

24. Grimaldi D, Agosti D: A formicine in New Jersey Cretaceous amber (Hymenoptera: Formicidae) and early evolution of the ants. Proc Natl Acad Sci 2001, 97:13678-13683.

25. Moreau CS, Bell CD, Vila R, Archibald SB, Pierce NE: Phylogeny of the ants: Diversification in the Age of Angiosperms. Science 2006, 312:101-104.

26. Yao Y-Z, Cai W-Z, Ren D: The Fossil Heteroptera of China: a review of present knowledge. Acta Zootaxon Sin 2004, 29:33-37.

27. Maere S, De Bodt S, Raes J, Casneuf T, Van Montagu M, Kuiper M, Van De Peer Y: Modeling gene and genome duplications in eukaryotes. Proc Natl Acad Sci 2005, 102:5454-5459.

28. Fan C, Xiang Q-Y: Phylogenetic analyses of Cornales based on 26S rRNA and combined 26S rDNA-matK-rbcL sequence data. Am J Bot 2003, 90:1357-1372

29. Eyde RH: Comprehending Cornus - puzzles and progress in the systematics of the dogwoods. Bot Rev 1988, 54:233-351.

30. Takhtajan AL: Diversity and Classification of Flowering Plants. New York, New York, USA: Columbia University Press; 1997.

31. David CT, Michael JD: Phylogeny and phylogenetic nomenclature of the Campanulidae based on an expanded sample of genes and taxa. Syst Bot 2010, 35:25-441. 
32. Ning GG, Xiao X, Lv HY, Li X, Zuo Y, Bao MZ: Shortening tobacco life-cycle accelerates functional gene identification in genomic research. Plant Biol 2012, 14:934-943.

33. Li R, Li Y, Kristiansen K, Wang J: SOAP: short oligonucleotide alignment program. Bioinformatics 2008, 24:713-714

34. Altschul SF, Madden TL, Schaffer AA, Zhang J, Zhang Z, Miller W, Lipman DJ: Gapped BLAST and PSI-BLAST: a new generation of protein database search programs. Nucleic Acids Res 1997, 25:3389-3402.

35. Rice $P$, Longden I, Bleasby A: EMBOSS: the european molecular biology open software suite. Trends Genet 2000, 16:276-277.

36. Conesa A, Gotz S, Garcia-Gomez JM, Terol J, Talon M, Robles M: Blast2GO: A universal tool for annotation, visualization and analysis in functional genomics research. Bioinformatics 2005, 21:3674-3676.

37. Kanehisa M, Araki M, Goto S, Hattori M, Hirakawa M, Itoh M, Katayama T, Kawashima S, Okuda S, Tokimatsu T, Yamanishi Y: KEGG for linking genomes to life and the environment. Nucleic Acids Res 2008, 36:D480-D484.

38. Edgar RC: MUSCLE: multiple sequence alignment with high accuracy and high throughput. Nucleic Acids Res 2004, 32:1792-1797.

39. Wernersson R, Pedersen AG: RevTrans - Constructing alignments of coding DNA from aligned amino acid sequences. Nucleic Acids Res 2003, 31:3537-3539.

40. Posada D, Crandall KA: MODELTEST: testing the model of DNA substitution. Bioinformatics 1998, 14:817-818.

41. Guindon S, Dufayard JF, Lefort V, Anisimova M, Hordijk W, Gascuel O: New algorithms and methods to estimate maximum-likelihood phylogenies: assessing the performance of PhyML 3.0. Syst Biol 2010, 59:307-321.

42. Swofford DL: PAUP*: Phylogenetic Analysis Using Parsimony ( ${ }^{*}$ and other Methods), ver 4.0. Sunderland MA: Sinauer Associates; 2003.

doi:10.1186/1756-0500-7-402

Cite this article as: Sun et al: Biological pattern and transcriptomic exploration and phylogenetic analysis in the odd floral architecture tree: Helwingia willd. BMC Research Notes 2014 7:402.

\section{Submit your next manuscript to BioMed Central and take full advantage of:}

- Convenient online submission

- Thorough peer review

- No space constraints or color figure charges

- Immediate publication on acceptance

- Inclusion in PubMed, CAS, Scopus and Google Scholar

- Research which is freely available for redistribution 